\title{
Espasmo hemifacial como presentación clínica de meningiomas intracraneales. Presentación de 3 casos y revisión de la literatura
}

\author{
L.F. Gómez-Perals; M. Ortega-Martínez; I. Fernández-Portales y J.M. Cabezudo-Artero
}

Servicio de Neurocirugía. Hospital Regional Universitario Infanta Cristina. Badajoz.

\section{Resumen}

El espasmo hemifacial (EH) constituye una entidad clínica caracterizada por contracciones tónico-clónicas de carácter involuntario de la musculatura inervada por el nervio facial. Como forma de presentación de meningiomas intracraneales es excepcional. Presentamos tres casos de pacientes con meningiomas cuya presentación clínica fue en forma de $\mathrm{EH}$, bien de forma aislada o asociado a sintomatología de hipertensión intracraneal o déficit neurológico focal. Se revisa la literatura y se discuten los posibles mecanismos fisiopatológicos que puedan intervenir en esta asociación.

PALABRAS CLAVE: Meningioma. Espasmo hemifacial.

Hemifacial spasm as clinical presentation of intracranial meningiomas. Report of three cases and review of the literature

\section{Summary}

Hemifacial spasm (HFS) is a clinical entity consisting of brief clonic jerking movements of the facial musculature, beginning in the orbicularis oculi with downward spreading to other facial muscles. Apart from vascular loop compression at the root exit zone of the facial nerve, other causes of HFS are rare. It is excepcional as a form of presentation of intracranial meningiomas We report three cases of patients with meningiomas who presented with HFS, either as an isolated sign or associated with symptoms of rise intracranial pressure or focal déficit. We review the literature and discuss the possible physiopathological mechanisms responsible for this association.

KEY WORDS: Meningioma. Hemifacial spasm.

Recibido: 17-02-04. Aceptado: 27-04-04
Introducción

El espasmo hemifacial (EH) es una entidad clínica caracterizada por contracciones tónico-clónicas de carácter involuntario de la musculatura inervada por el nervio facial. La etiología más frecuente es la compresión de la "root exit zone" (REZ) del nervio facial por un asa vascular. Como signo de presentación, único o asociado a otra sintomatología, de meningiomas intracraneales es excepcional. Presentamos tres casos en los que encontramos esta asociación. Se revisa la literatura y se discuten los posibles mecanismos fisiopatológicos responsables de esta asociación.

\section{Casos clínicos}

Caso 1. Varón de 65 años de edad, con antecedentes de diabetes mellitus no insulindependiente, con clínica de EH de varios años de evolución, mareos, cefaleas, pérdida de memoria y cambios de carácter de 6 meses de evolución e incontinencia urinaria en el mes previo a su ingreso. El examen neurológico resultó normal, excepto por su EH. La tomografía computarizada (TC) cerebral mostró la presencia de un proceso expansivo frontal izquierdo de $6,5 \times 5 \times$ $6,5 \mathrm{cms}$ de diámetro en los ejes anteroposterior, lateromedial y craneocaudal respectivamente, con captación intensa y distribución homogénea de contraste y edema perilesional con importante efecto masa sobre estructuras vecinas (Figura 1). La angiografía carotídea izquierda reveló un desplazamiento contralateral de la cerebral anterior sin teñido tumoral evidente. Se intervino quirúrgicamente practicándose craneotomía bifrontal y exéresis completa de un meningioma de la región anterior de la hoz cerebral cuyo tipo histopatológico fue meningotelial. En el día $7^{\circ}$ del postoperatorio, siendo la evolución hasta entonces óptima, con mejoría de su sintomatología de $\mathrm{EH}$, sufrió un cuadro agudo de asfixia asociado a su movilización,

Abreviaturas. APC: ángulo pontocerebeloso. EH: espasmo hemifacial. HFS: hemifacial spasm. REZ: root exit zone. TEP: Tromboembolismo pulmonar. 

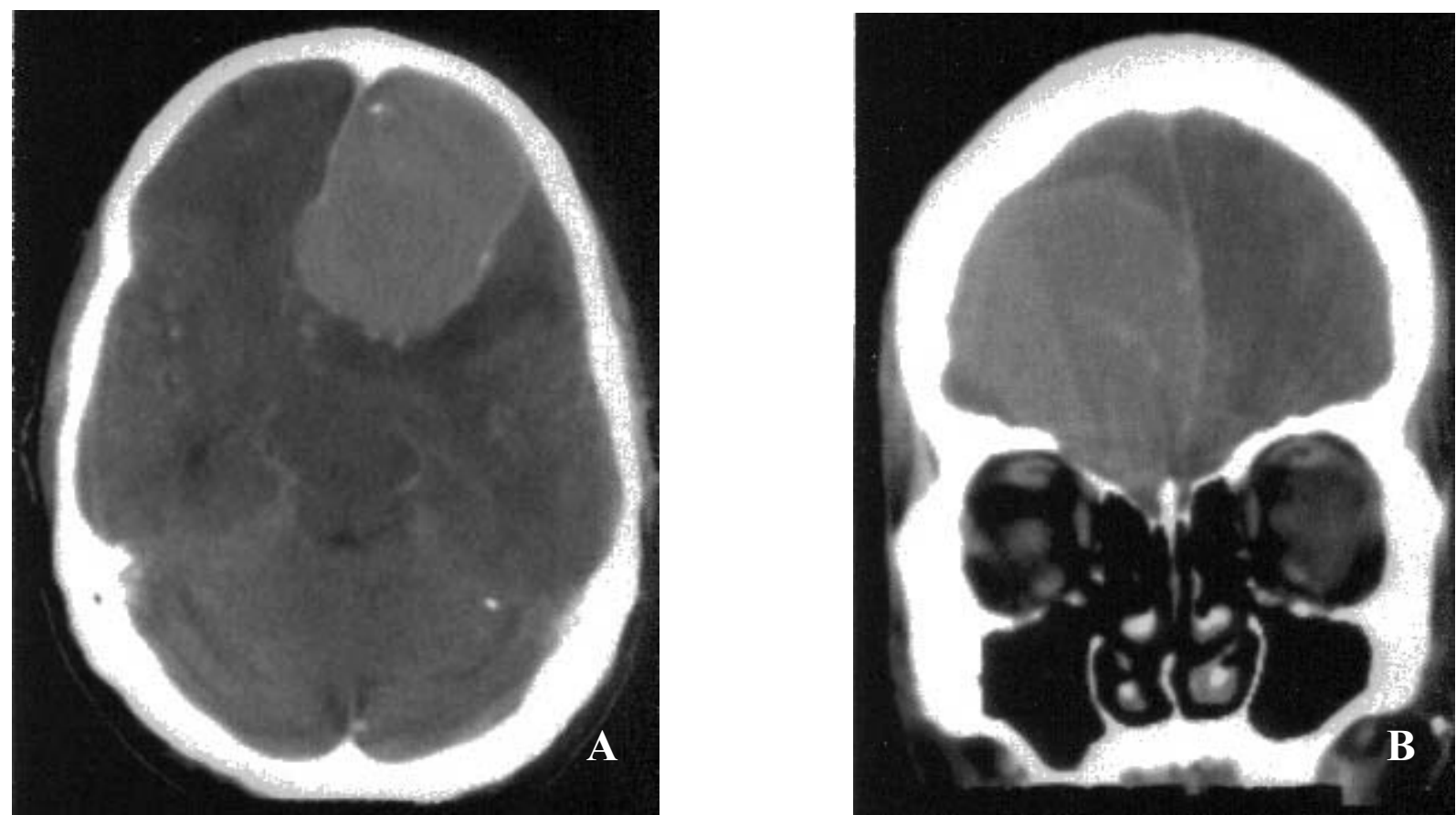

Figura 1. CASO 1. Varón, 65 años. Meningioma meningotelial de la hoz cerebral frontal izquierda de 6,5 x $5 \times 6,5 \mathrm{cms}$ de tamaño. Clínica de varios años de EH contralateral. Exitus postquirúrgico por TEP. TC cerebral. A. Corte axial con contraste. Se observa el realce intenso de la tumoración y la distribución homogénea del contraste, así como edema perilesional importante con efecto masa sobre estructuras vecinas y en dirección al tronco cerebral. B. Corte coronal con contraste.

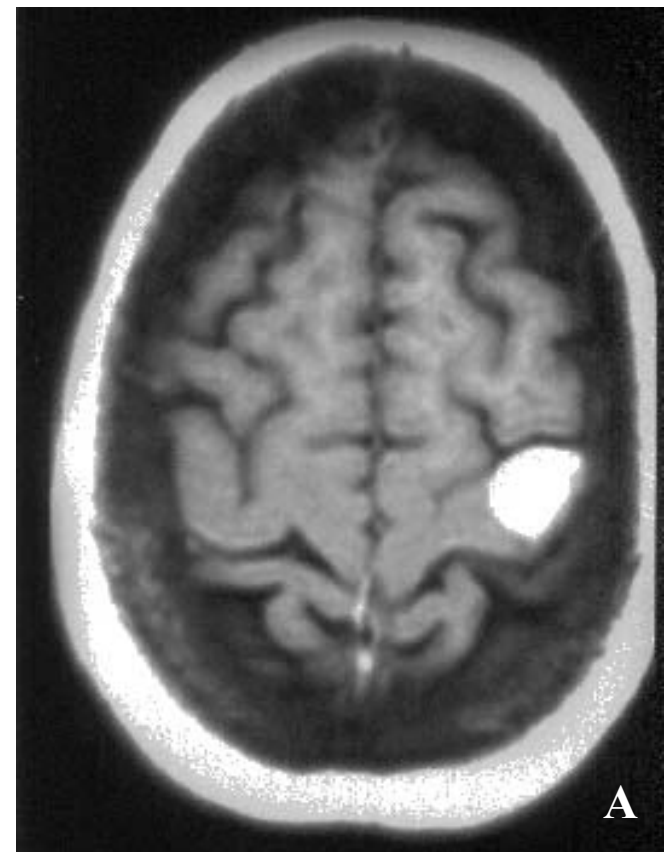

probablemente debido a un episodio de tromboembolismo pulmonar (TEP) masivo -no fue posible practicar autopsia por negación de los familiares-, siendo exitus.

CASO 2. Mujer de 44 años de edad, sin antecedentes de interés, con historia de 2 años de evolución consistente en

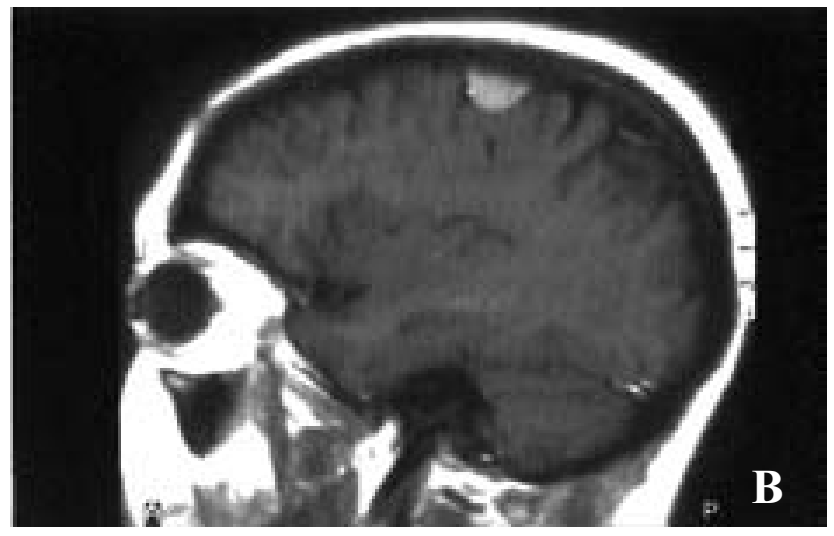

Figura 2. CASO 2. Mujer, 44 años. Meningioma transicional del girus frontal ascendente izquierdo (región facial del área motora) de unos $2 \mathrm{cms}$ de tamaño. Dos años de EH contralateral. Mejoría postquirúrgica parcial. RMN cerebral con gadolinio. A. Corte axial. B. Corte sagital.

episodios repetitivos de cierre ocular derecho involuntario, en ocasiones continuo y otras veces en forma de sacudidas de varios minutos de duración, asociado a sensación de tirantez en mejilla derecha con ocasional desviación de la comisura bucal a la derecha en forma de espasmos breves que en ocasiones podían ser muy intensos y asociarse a un zumbido continuo en oído derecho. El examen neurológico 


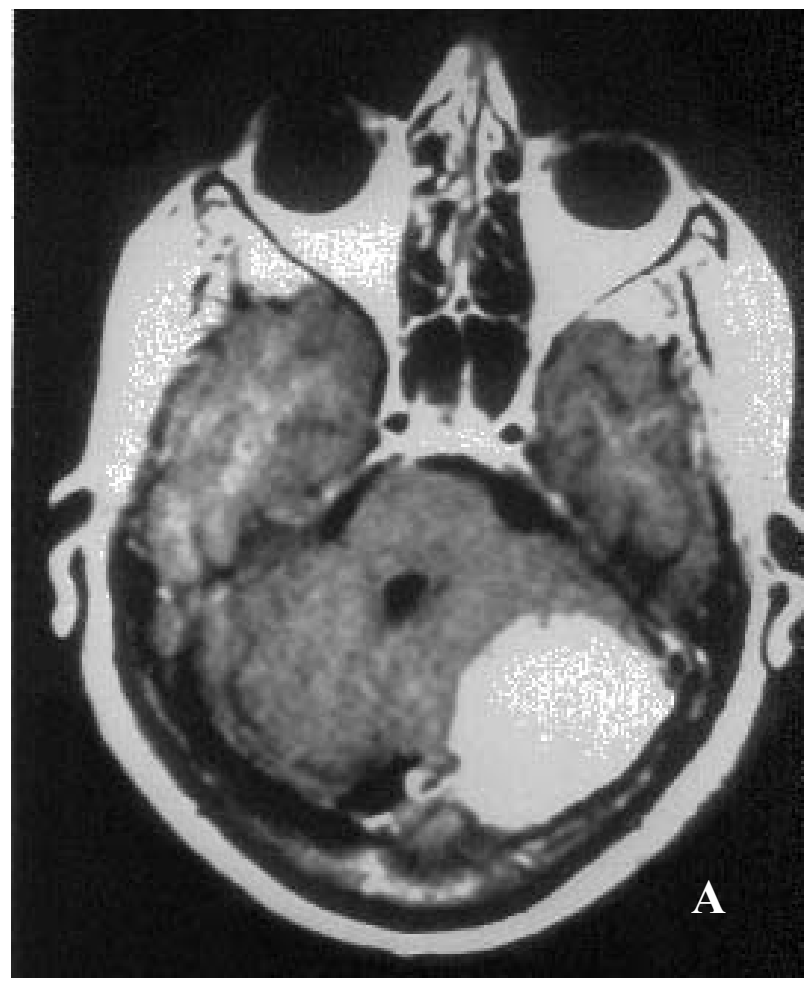

mostró un cuadro típico de EH siendo el resto de la exploración normal. La resonancia magnética nuclear (RMN) cerebral reveló la presencia de una lesión de unos $2 \mathrm{cms}$ de tamaño localizada en la circunvolución frontal ascendente, afectando probablemente a la región facial del área motora, con amplia base de implantación en duramadre y captación intensa y homogénea de contraste (Figura 2). El estudio neurofisiológico del nervio facial fue normal y el electroencefalograma mostró un foco de ondas lentas frontal izquierdo. Se practicó una craneotomía frontoparietal izquierda y resección completa de un meningioma de convexidad cuyo tipo histopatológico fue transicional. El postoperatorio cursó sin complicaciones, siendo la evolución de su EH errática, precisando tratamiento con toxina botulínica.

CASO 3. Mujer de 65 años, sin antecedentes de interés, con cuadro de 15 meses de evolución de $\mathrm{EH}$ asociado en los últimos 4 meses a cefaleas y dificultad visual. El examen neurológico mostró un cuadró típico de EH y una disminución de la agudeza visual sin edema de papila en el fondo de ojo. La RMN reveló la presencia de un proceso expansivo de fosa posterior de $5 \mathrm{cms}$ de diámetro mayor, con efecto masa y desplazamiento del IV ventrículo -aunque sin provocar hidrocefalia-, con captación intensa y distribución homogénea de contraste (Figura 3). Se realizó craniectomía suboccipital y extirpación completa de un meningioma insertado en la duramadre de la convexidad cerebelosa izquierda, seno transverso y tentorio cuyo

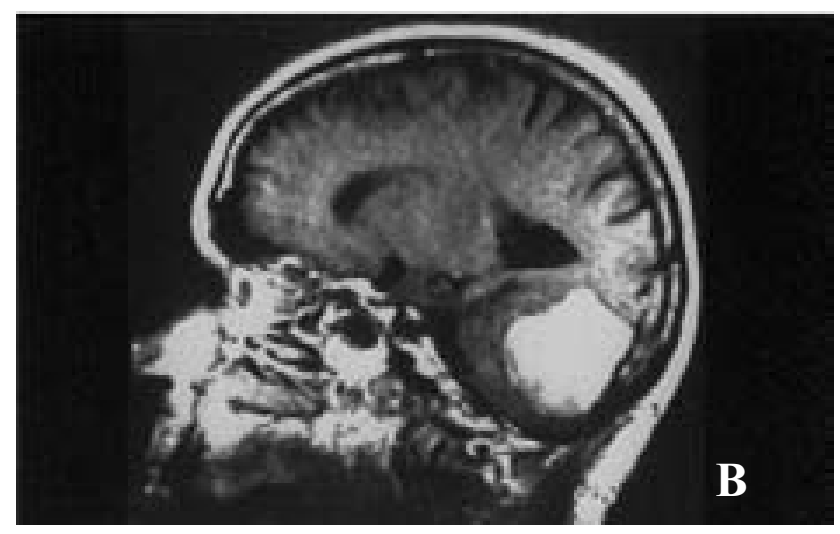

Figura 3. CASO 3. Mujer, 65 años. Meningioma psamomatoso de la convexidad cerebelosa izquierda, seno transverso y tentorio de $5 \mathrm{cms}$ de tamaño. Quince meses de EH ipsilateral. Mejoría postquirúrgica leve. RMN cerebral con gadolinio. A. Corte axial. B. Corte sagital.

tipo histopatológico fue psamomatoso. El postoperatorio cursó sin complicaciones. Tras una mejoría postquirúrgica parcial, la evolución de su EH fue alternando períodos de agravamiento de su sintomatología con épocas de mejoría, requiriendo la administración de toxina botulínica para un mejor control -y finalmente remisión- de su EH. Tras 11 años de seguimiento la paciente está asintomática.

\section{Discusión}

Existen varios tipos de trastornos focales del movimiento que afectan a la musculatura facial, provocando contracciones anormales espontáneas e involuntarias. Wilkins $^{12}$, en su magnífica revisión, describe, de forma resumida, los más frecuentes y de forma más amplia el $\mathrm{EH}$, aportando abundante bibliografía -367 citas-, lo que le convierte en una revisión de recomendada, por no decir obligada, lectura.

El EH, más frecuente en el sexo femenino y en la edad adulta, se suele presentar de forma insidiosa, afectando a la musculatura orbicular en forma de fasciculaciones del párpado, extendiéndose posteriormente al resto de la musculatura facial ipsilateral. Los espasmos cursan de forma paroxística y se incrementan en frecuencia e intensidad con el paso del tiempo. Las contracciones mantenidas pueden durar hasta un minuto. Los factores emocionales o la fatiga psíquica o física pueden agravar la sintomatología. El tratamiento puede ser médico -la inyección de toxina botulínica puede producir un alivio rápido y duradero- o quirúrgico -lesiones o arterias que produzcan compresión del nervio facial.

Nuestra revisión de la literatura se resume en la tabla 1. Hemos encontrado un total de 16 casos publicados, de los cuales 12 tuvieron una localización infratentorial -9 de 
Tabla I

Revisión bibliográfica

\begin{tabular}{|c|c|c|c|c|c|}
\hline \multirow[t]{2}{*}{ AÑo } & \multirow{2}{*}{ AUTORES (Referencia bibliográfica) } & \multirow[t]{2}{*}{$\mathbf{N}^{\circ}$ CASOS } & \multicolumn{2}{|c|}{ INFRATENTORIAL } & \multirow[t]{2}{*}{ SUPRATENTORIAI } \\
\hline & & & APC & Otras localizaciones & \\
\hline 2003 & Zhao, W.G. et al. ${ }^{14}$ & 1 & 1 & & \\
\hline 2003 & Wrobel-Wisniewska, G. et al..$^{13}$ & 1 & & 1 & \\
\hline 2001 & Galvez-Jiménez, N. et al. ${ }^{4}$ & 1 & 1 & & \\
\hline 2001 & Iwai, Y. et al. ${ }^{5}$ & 2 & 2 & & \\
\hline 1996 & Bhayani, R. et al. ${ }^{2}$ & 1 & & & 1 \\
\hline 1995 & Ogasawara, H. et al. ${ }^{9}$ & 1 & & 1 & \\
\hline 1995 & Rhee, B.A. et al. ${ }^{10}$ & 1 & 1 (contralateral) & & \\
\hline 1992 & Nagata, S. et al. ${ }^{8}$ & 2 & 1 & 1 & \\
\hline 1991 & Lin, P.J. et al. ${ }^{6}$ & 1 & 1 & & \\
\hline 1991 & Wilkins, R.H. ${ }^{12}$ & 1 & 1 & & \\
\hline 1990 & Toyota, A. et al. ${ }^{11}$ & 1 & & & 1 \\
\hline 1989 & Chang, C.G. et al. ${ }^{3}$ & 2 & No especifica & No especifica & No especifica \\
\hline 1987 & Miyazaki, S. et al. ${ }^{7}$ & 1 & 1 & & \\
\hline & Revisión bibliográfica & 16 & 9 & 3 & 2 \\
\hline
\end{tabular}

ellos en ángulo pontocerebeloso (APC) (8 ipsilateral ${ }^{4,5,6,7,8,14}$ y 1 contralateral al $\mathrm{EH}^{10}$ ) y 3 casos en otras localizaciones de la fosa posterior (FP) ${ }^{8,9.13}$. Únicamente en 2 casos la localización fue supratentorial ${ }^{2,11}$. Por último, en 2 casos no hemos encontrado la descripción de la localización de los meningiomas ${ }^{3}$.

En cuanto a la fisiopatología, el EH se produce por la afectación del VII par craneal por una estructura anatómica, que se puede localizar a cualquier nivel entre el núcleo del facial y el foramen estilomastoideo pero que más específicamente se localiza en proximidad a la REZ. Por ello, resulta muy difícil atribuir al EH una etiología supranuclear, bien sea supratentorial o infratentorial, distinta del ángulo pontocerebeloso. En estos casos, el EH se atribuye a un mecanismo de distorsión y desplazamiento del tallo cerebral que, conllevarían una compresión neurovascular secundaria del nervio facial por un "efecto remoto". Así, Rhee y col. ${ }^{10}$ describen un caso único de meningioma del APC asociado a EH contralateral y ofrece dos explicaciones a este hallazgo: el mencionado mecanismo de distorsión-compresión-rotación troncoencefálica, con la consiguiente afectación del facial a nivel de la REZ, o la posible compresión del facial entre el tronco y estructuras adyacentes de mayor consistencia como el peñasco o la incisura tentorial.

Este mecanismo de distorsión troncoencefálica, con la consecuente compresión del nervio facial, resulta aún más difícil de aceptar en los casos de localización supratentorial. Sólo Bhayani y col. $^{2}$-meningioma de la hoz a nivel occipital - y Toyota y col. ${ }^{11}$-meningioma de la unión falcotentorial y región pineal- presentan casos de localización supratentorial. El primer autor atribuye el EH a la distorsión anatómica de los elementos de la fosa posterior por un mecanismo parecido al "efecto remoto" aludido anteriormente, de forma que el desplazamiento caudal del tentorio pudiera haber alterado la REZ del VII par craneal.

Con respecto a nuestra casuística, en el caso 1 -meningioma de la hoz frontal de gran tamaño- el mecanismo responsable podría haber sido el efecto masa ejercido por la tumoración -que se aprecia sobretodo en la figura 2 Bcreándose un cono de presión transtentorial trasmitido a las estructuras de fosa posterior con distorsión troncoencefálica y consecuente afectación del facial.

En el caso 3 -localización infratentorial distinta al APCcreemos que el EH podría atribuirse a la distorsión de las estructuras de la FP con compresión secundaria del nervio facial.

Por último, en el caso 2 -localización supratentorial en 
proximidad al área motora-, la ausencia de efecto masa descarta la posibilidad de un mecanismo de distorsión y desplazamiento del tallo cerebral y no hemos encontrado argumentos para explicar que la afectación de la vía motora supranuclear pueda explicar la presencia de EH. Sin embargo, aunque la explicación más plausible pudiera ser la de una asociación casual, no hay que olvidar la extraordinaria rareza de una presentación de este tipo, dado que la frecuencia anual de meningiomas viene a ser de 2 casos por 100.000 habitantes y año y la prevalencia de EH pueda estar en 14,5 por 100.000 en mujeres y 7,4 por 100.000 en hombres aproximadamente ${ }^{1}$.

Por último, algunos autores describen un alivio importante del EH tras la cirugía $a^{5,7,8,9,11,13}$ mientras que otros no hacen mención de la evolución clínica. En nuestro caso 1, aunque hubo mejoría postoperatoria, el seguimiento se vio truncado por un episodio de TEP masivo con exitus. En nuestros casos 2 y 3 hubo mejoría aunque de forma parcial, siendo necesaria la administración de toxina botulínica para un mejor control, lo que en el caso 3 pudiera atribuirse a una distorsión permanente o residual del facial a pesar de la descompresión quirúrgica.

\section{Conclusión}

El HE como forma de presentación de meningiomas es un hecho extraordinariamente infrecuente. Presentamos 3 casos en los que se dio esta asociación. El mecanismo fisiopatológico responsable creemos pudiera ser debido al efecto masa de la tumoración con distorsión y desplazamiento del tallo cerebral que, conllevarían una compresión neurovascular secundaria del nervio facial por un "efecto remoto". En uno de nuestros casos no encontramos una explicación satisfactoria.

\section{Bibliografía}

1. Auger, R.G., Whisnant, J.P.: Hemifacial spasm in Rochester and Olmsted County, Minnesota, 1960 to 1984. Arch Neurol 1990; 47: 1233 - 1234.

2. Bhayani, R., Goel, A.: Occipital falcine meningioma presenting with ipsilateral hemifacial spasm: a case report. $\mathrm{Br}$ J Neurosurg 1996; 10: 603-5.

3. Chang, C.G., Cho, D.Y., Lee, J.C., Yang, D.Y.: Intra- cranial meningiomas - 5 year analysis. [Artículo en chino]. Zhonghua Yi Xue Za Zhi (Taipei) 1989; 43: 321-30.

4. Galvez-Jimenez, N., Hanson, M.R., Desai, M.: Unusual causes of hemifacial spasm. Semin Neurol 2001; 21: 75-83.

5. Iwai, Y., Yamanaka, K., Nakajima, H.: Hemifacial spasm due to cerebellopontine angle meningiomas - two cases reports. Neurol Med Chir (Tokyo) 2001; 41: 8789.

6. Lin, P.J., Li, C.S., Chang, G.S.: Experiences in the management of cerebellopontine angle tumour: an analysis of 64 cases. Gaoxiong Yi Xue Ke Xue Za Zhi 1991; 7: 329-33.

7. Miyazaki, S., Fukushima, T., Nagai, A., Tamagawa, T.: Cerebellopontine angle meningioma presenting as hemifacial spasm. No Shinkei Geka 1987; 15: 683-6

8. Nagata, S., Matsushima, T., Fujii, K., Fukui, M., Kuromatsu, C.: Hemifacial spasm due to tumor, aneurysm, or arteriovenous malformation. Surg Neurol 1992; 38: 204-9.

9. Ogasawara, H., Oki, S., Kohno, H., Hibino, S., Ito, Y.: Tentorial meningioma and painful tic convulsif. Case report. J Neurosurg 1995; 82: 895-7.

10. Rhee, B.A., Kim, T.S., Kim, G.K., Leem, W.L.: Hemifacial spasm caused by contralateral cerebellopontine angle meningioma: case report. Neurosurgery 1995; 36: 393-5.

11. Toyota, A., Takahashi, A., Yoshida, Y., Murakami, T., Saiki, L, Kanaya, H.: Meningioma of pineal region. [Artículo en japonés]. No Shinkei Geka. 1990; 18: 745-9.

12.Wilkins, R.H.: Hemifacial spasm: A review. Surg Neurol 1991; 36: 251- 277.

13. Wrobel-Wisniewska, G., Kasprzak, P., Zawirski, M.: [In Process Citation]. [Artículo en polaco]. Neurol Neurochir Pol. 2003; 37: 459-63.

14. Zhao, W.G., Zhu, J., Shen, J.K., Hu, J.Q., Tan, L., Miu, F., Chen, K.M.: Preoperative etiological diagnosis of hemifacial spasm: a comparative study between magnetic resonante tomographic angiography and intra-operative findings. [Artículo en chino]. Zhonghua Yi Xue Za Zhi. 2003; 83: 1871-3.

Gómez-Perals, L.F.; Ortega-Martínez, M.; FernándezPortales, I,; Cabezudo-Artero, J.M.: Espasmo hemifacial como presentación clínica de meningiomas intracraneales. Presentación de 3 casos y revisión de la literatura. Neurocirugía 2005; 16: 21-26.

Correspondencia postal: Luis Gómez Perals. Apartado de correos 672. 06080 Badajoz. 
Comentario al trabajo: Espasmo hemifacial como presentación clínica de meningiomas intracraneales. Presentación de tres casos y revisión de la literatura de Gómez-Perals y cols.

Los autores presentan tres casos de meningiomas intracraneales asociados clínicamente a espasmo hemifacial $(\mathrm{EH})$, y estudian la posible relación de causalidad de la lesión tumoral sobre el EH. Así, para los casos 1 y 3 (un gran meningioma frontobasal izquierdo y un meningioma cerebeloso izquierdo), los autores proponen un mecanismo de distorsión anatómica y compresión neurovascular por efecto remoto, mientras que para el caso 2 (meningioma rolándico izquierdo), no encuentran una relación causal satisfactoria.

El EH es una entidad clínica bien conocida secundaria habitualmente a una microcompresión vascular de la REZ del nervio facial, típicamente a expensas de la AICA, si bien otras estructuras vasculares pueden estar implicadas. El EH secundario a otras lesiones estructurales que causan una distorsión directa o próxima es un hecho infrecuente pero bien conocido. Tal como comentan los autores, los casos secundarios a microcompresión por lesiones remotas son extremadamente infrecuentes y no siempre han sido explicados suficientemente.

Algunas cuestiones quedan abiertas tras leer el artículo y antes de asumir una posible relación causa-efecto en los casos descritos de meningioma-EH. No nos centraremos en el caso 2, ya que como señalan los autores, no es posible hallar una relación causal satisfactoria. Sin embargo, sí merece la pena señalar que la imagen de RM craneal parece localizar la lesión tumoral sensiblemente más próxima a la cisura interhemisférca que a la silviana, en un área que según el homúnculo de Penfield se centra sobre los grupos motores del miembro superior derecho.

Respecto a los casos 1 y 3 cabe preguntarse en primer lugar cuál fue la secuencia temporal entre el EH y el meningioma. El hecho de que el EH preceda entre uno y varios años a la clínica asociada a hipertensión intracraneal obligaría metodológicamente a confirmar la presencia del meningioma en el momento del diagnóstico del EH, excluyendo así la posibilidad de que el EH precediera al desarrollo tumoral. Podemos preguntarnos adicionalmente si no deberíamos considerar la hipertensión intracraneal como forma de presentación de estos tumores, siendo el EH un fenómeno asociado.

En segundo lugar, resulta llamativo en el caso 1 que una herniación central produzca una distorsión anatómica remota sobre el VII par durante años sin otra clínica acompañante de hipertensión intracraneal, como cefalea, disminución del nivel de conciencia o lesión de otros pares craneales más susceptibles a los estados de hipertensión intracraneal como es el VI par.

Respecto a la coherencia con los mecanismos de microcompresión vascular descritos por otros autores, podemos preguntarnos si los estudios preoperatorios de imagen o los hallazgos quirúrgicos en el caso 3 mostraron alguna prueba de microcompresión del VII par. Las técnicas de RM de alta resolución permiten localizar el vaso responsable de la microcompresión en un porcentaje cada vez mayor de pacientes. En los casos de meningioma-EH que documentan los autores, parece deseable disponer no sólo de la imagen de la lesión tumoral, sino también del área clínicamente elocuente y diana de las hipótesis etiológicas, la REZ del VII par.

Por último, respecto a la evolución posquirúrgica del $\mathrm{EH}$, la mayoría de los autores hablan de tasas de control de la enfermedad próximas al 90\%. El paciente del caso 1 parece evolucionar favorableinente, si bien un exitus precoz imposibilita confirmar la persistencia de dicha mejoría. En los casos 2 y 3 en que se describe una mejoria parcial y errática, cabe preguntarse si estaría indicado repetir un estudio de RM centrado en la fosa posterior y en el VII par, e incluso si se planteó una nueva intervención encaminada a optimizar la microdescompresión del REZ del facial.

Se trata en definitiva de la presentación de tres casos interesantes de asociación entre hemiespasmo facial y meningioma intracraneal que despierta el ánimo de profundizar en el conocilpiento de la fisiopatología del EH antes de aceptar una posible relación causal.

P. Miranda
R.D. Lobato
Madrid

